\title{
L’Algérie vers un Développement Agricole Durable
}

\author{
Attalah Nadhira* \\ Bouchama Ouahiba**
}

\section{RÉSUMÉ}

Ce document de recherche met en évidence la situation désastreuse du secteur agricole dans un pays agricole tel que l'Algérie, qui ne peut pas garantie son autosuffisance alimentaire. Les importations alimentaires ont atteint 10,27 milliards de dollars en 2013, la part du lion des céréales était de 3,54 milliards de dollars en 2014. En 2015, la facture d'importation a augmenté de 7,3\%. Nous avons donc noté que les différentes politiques adoptées par l'Algérie depuis l'indépendance n'ont pas été en mesure de garantir l'indépendance alimentaire, ce qui a des conséquences désastreuses pour le pays : l'Algérie s'est retrouvée confrontée à des défis majeurs pour le développement agricole.

A travers cette recherche, nous avons voulu mettre en évidence les résultats des précédentes politiques agricoles et la réalité du développement agricole durable. [Bul. Soc. Géog. d'Égypte, 2018, 91: 49-66]

Mots-clés: l'Algérie, développement du secteur agricole, développement durable, sécurité alimentaire.

\section{Introduction}

Suite aux politiques ratées adoptées par l'Algérie depuis l'indépendance, à l'augmentation du déficit alimentaire, à la hausse des importations et à la baisse des prix du pétrole, l'Algérie a cherché des solutions pour améliorer son économie et sa sécurité alimentaire. Grâce à la mise en œuvre du Programme National de Développement Agricole et de la Politique de Renouveau Rural et Agricole, en 2008, le développement de l'agriculture était à la disposition de 200 milliards de dinars / an.

Cette approche a également imposé en raison des exigences des procédures qui ont été publiées dans le cadre des négociations sur l'adhésion de l'Algérie à l'Organisation mondiale du commerce et mis en œuvre depuis 2005. Accord Union avec l'Union européenne.

Le secteur agricole algérien a connu trois étapes depuis l'indépendance et a subi une série de réformes liées à différents titres: autogestion, révolution

* Maître de conférences-B- École Normale Supérieure Bouzaréah - Alger.

** Maître de conférences-B- École Normale Supérieure Bouzaréah - Alger. 
agricole, restructuration, de nombreux domaines tels que l'immobilier, le crédit, la finance et la commercialisation ont été positifs pour l'environnement économique et social. Ils n'abandonnent pas les négatifs, car malgré les similitudes d'objectifs, mais l'impact était différent, chaque politique a subi des changements partiels du secteur agricole.

Après la détérioration du secteur agricole dans la dernière décennie du siècle dernier et la poursuite du développement du secteur agricole, l'Algérie s'est engagée dans une nouvelle approche et une nouvelle politique avec le plan national de développement agricole et lui a donné une dimension rurale. Renouveau agricole et rural en 2008 et programme quinquennal 2010-2014 Ces projets s'inscrivent dans le cadre des efforts de l'État pour parvenir à un développement agricole durable.

\section{La question qui se pose: est ce que les politiques menées par} l'Algérie sont suffisantes pour parvenir à un développement agricole durable?

\section{1- Situation géographique de l'Algérie:}

L'Algérie est située sur la rive sud de la méditerranée entre $18^{\circ}$ et $38^{\circ}$ de l'altitude nord et entre $9^{\circ}$ de longitude ouest et $12^{\circ}$ de longitude. Limitée à l'Est par la Tunisie et la Libye .au sud par le Niger et le Mali au sud ouest par la Mauritanie et le Sahara occidental et à l'ouest par le Maroc, avec une superficie de $2381.741 \mathrm{Km} 2{ }^{2}$ le plus grand pays d'Afrique. L'Algérie est caractérisée par un contraste physique et climatique bien marqué entre les régions nord et sud, au nord le relief est souvent accidenté, au sud le Sahara occupe $85 \%$ de cette étendu Deux chaînes montagneuses importantes, l'Atlas Tellien au Nord et l'Atlas Saharien au Sud, séparent le pays en trois types de milieux qui se distinguent par leur relief et leur morphologie, donnant lieu à une importante diversité biologique. On distingue du Nord au Sud, le Système Tellien, les Hautes Plaines steppiques et le Sahara (Voir la carte $n^{\circ} 01$ ).

L'Algérie comptait environ 40 millions d'habitants en juillet 2016 avec un taux de croissance annuel de $1,17 \%$.

\section{2- Climat et réseau hydrographique:}

Un climat méditerranéen couvre le Nord, tandis qu'un climat désertique règne sur le Sud, dont le Sahara est caractérisée par ses amplitudes thermiques qui sont considérables à cause des variations de températures et aussi extrêmement élevées pendant le jour et très basses durant la nuit. 


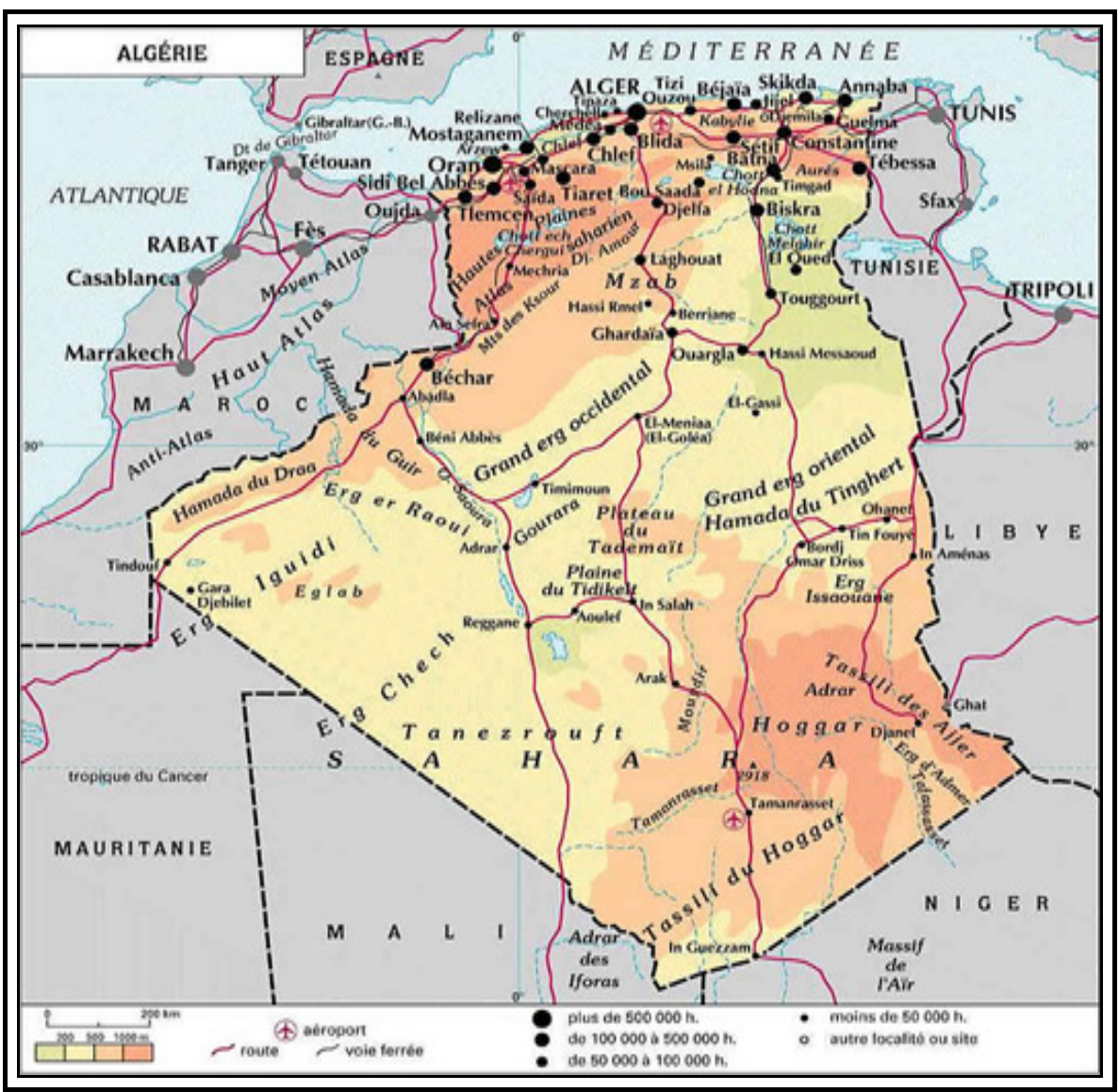

Carte n ${ }^{\circ} 1$

Concernant le réseau hydrographique, il existe quelques cours d'eau côtiers au centre ou à l'est du pays dont les plus fameux sont la Soummam, Medjerda, Sebou, Hami, Macta et Mazafran. Cependant le Chélif reste le plus long fleuve d'Algérie et sa longueur est de $725 \mathrm{~km}$. Ce fleuve est situé au nord-ouest de l'Algérie, il prend sa source dans l'Atlas tellien et se jette dans la Méditerranée. Il débite, dans les périodes de crues, $1500 \mathrm{~m} 3 / \mathrm{s}$. Au sud de la région du Tell, les cours d'eau ne sont pas permanents. Il existe de nombreux lacs dans les régions désertiques, mais ce sont des lacs temporaires et salés pour la majorité comme Chott ech Chergui et Chott el Hodna. Les courts d'eau du Tell se déversent dans la Méditerranée. Mais, ceux qui descendent vers l'Atlas saharien font partie de la plus grande réserve d'eau au monde. Ils forment une nappe phréatique dite la nappe de l'Albien. Cette nappe est la plus grande réserve au monde d'eau douce, elle est enfouie sous le sable du désert algérien et elle a une superficie 
de $900000 \mathrm{~km} 2$. Elle longe presque tout le Sahara algérien ce qui rend facile l'accès à l'eau pour les cinq pays voisins. (Voir la carte $n^{\circ} 02$ )

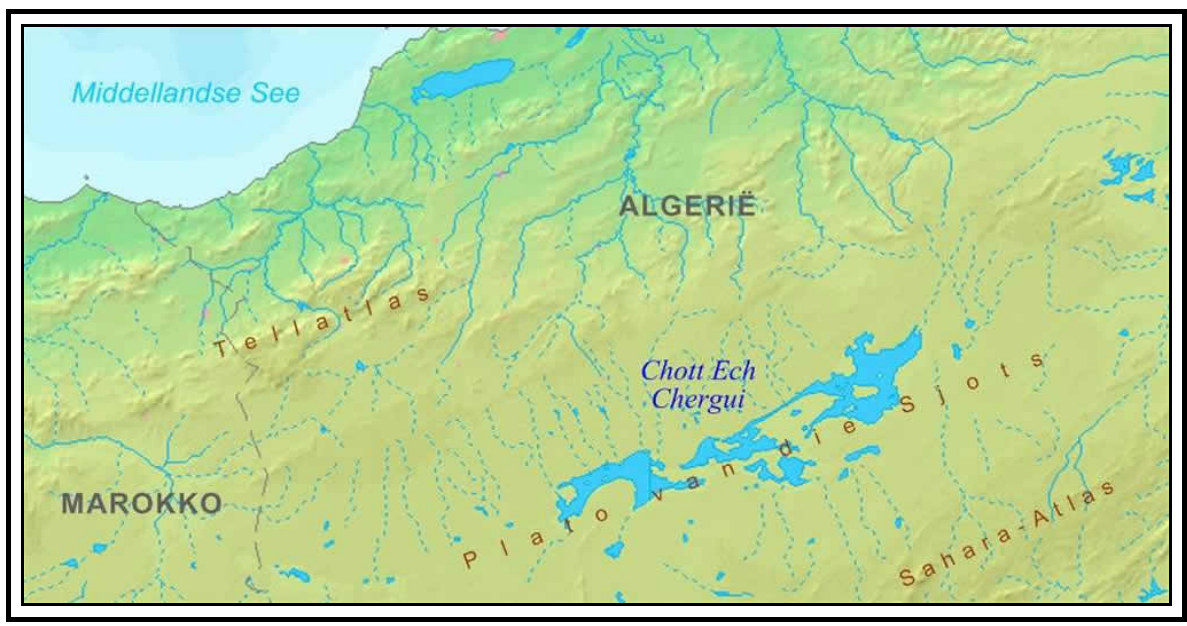

\section{Carte $\mathbf{n}^{\circ} \mathbf{0 2}$}

\section{3- Répartitions des terres agricoles:}

Les terres se répartissent de la façon suivante:

- Terres improductives estimées à 191 millions d'hectares.

- Terres forestières couvrant environ une superficie de 4,3 millions d'hectares.

- Les parcours et la steppe couvrent environ 34,3 millions d'hectares.

Les parcours et la Steppe Totale (SAT) couvre 8,2 millions d'hectares, dont environ 880000 hectares de terres sont non productives (bâtiments, chemins, etc. $)^{(1)}$ (voir la figure $\left.n^{\circ} 01\right)$.

\section{4- L'occupation des terres agricole:}

La superficie agricole totale représente trois pour cent de la superficie totale de l'Algérie comprenant:

- Cultures herbacées: 3,8 millions ha.

- Terres au repos (jachères): 3,7 millions ha.

- Plantations fruitières: 576990 ha.

- Vignobles: 81550 ha.

- Prairies naturelles: 23640 ha.

- terres improductives estimées à 191 millions d'hectares.

- Terres forestières couvrant environ une superficie de 4,3 millions $\mathrm{d}^{\prime}$ hectares ${ }^{(2)}$. (Voir la figure $\mathrm{n}^{\circ} 02$ ).

(1) Ministère de l'agriculture et de développement rural statistique année 2012.

(2) Ministère de l'agriculture et de développement rural statistique année 2012. 


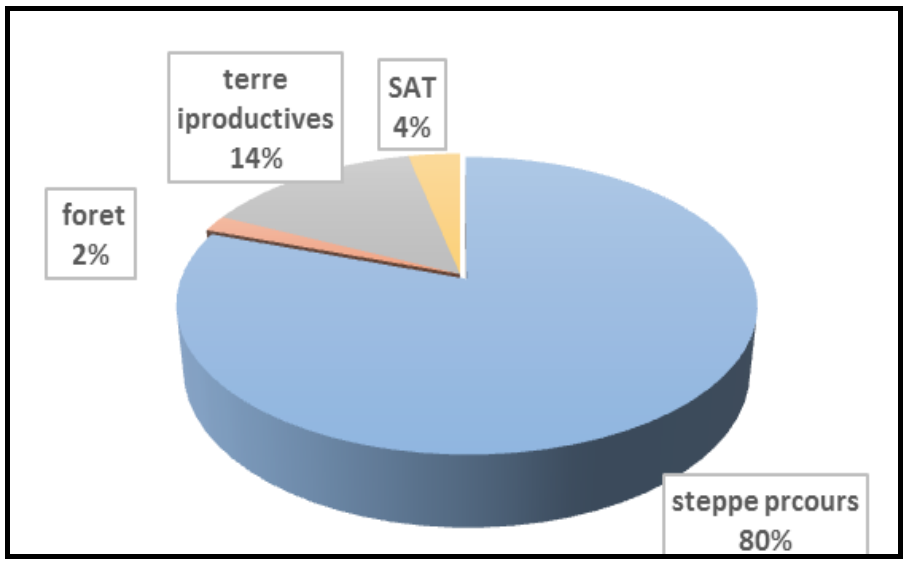

Figure $\mathbf{n}^{\circ} \mathbf{0 1}$

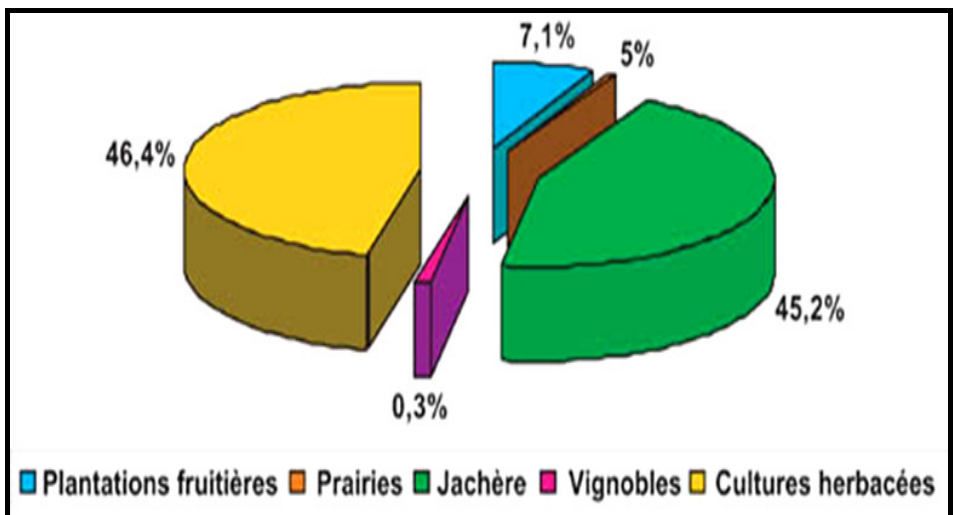

Figure $\mathbf{n}^{\circ} \mathbf{0 2}$

\section{5- Les différentes réformes du secteur agricole depuis l'indépendance:}

A l'évènement de l'indépendance, les responsables du pays étaient préoccupés à remettre de l'ordre dans la création du nouvel Etat. Ils ont été pris de court concernant le foncier agricole. Ils ont tout simplement laissé les terres à ceux qui se trouvaient à proximité, en l'occurrence les ouvriers agricoles qui travaillaient chez le colon. C'est ainsi que plus de 2 millions d'hectares de terres arables et environ 200000 ha de forêt se sont retrouvés, $\mathrm{du}$ jour au lendemain biens vacants et à la disposition des ouvriers qui occupaient l'espace rural ${ }^{(1)}$.

(1) BOURI Chaouki, CHENNOUF Sadok, MahmoudiOumeriem; Impacts de la politique de développement agricole et rural PNDA/PNDAR sur la relance économique enAlgérie; Université d'Oran. Les cahiers du MCAS N 08 Décembre 2012 p. 42.

\section{$-53-$}




\section{5-1- L'autogestion:}

Le transfert de propriété massif a commencé à se matérialiser par une intervention de l'Etat qui a promulgué l'Ordonnance 62-20 du 24 août 1962, relative à la protection et la gestion des biens vacants et le décret de mars 1963. Ces textes sont venus régulariser une situation de fait. Les hautes autorités du pays institutionnalisé ce qu'on a appelé l'autogestion ou système socialiste. C'est ainsi qu'en 1965, le secteur agricole autogéré s'étendait sur 2,3 millions ha, occupés par les colons durant la colonisation. L'Ordonnance 66-182 du 06 mai 1966, a dévolu à l'Etat la propriété des biens déclarés initialement vacants. Il en devient donc le seul propriétaire. ${ }^{(1)}$

\subsubsection{Le système autogéré se distingue par:}

- Un mode de production collectiviste. La notion de propriété privée est supprimée au profit de la propriété collective.

- La forme d'organisation est décentralisée. Les centres d'activités ont un développement autonome.

- L'objectif initial est de permettre un développement optimal dans ses dimensions politiques et économiques.

Très vite l'autogestion a montré ses limites. L'Etat a tenté d'y remédier en le consolidant par la parution de pas moins de 7 décrets dont le but était de réformer en profondeur un secteur agricole délabré.

La gestion centralisée et administrée à outrance de l'autogestion a été le fossoyeur de ce mode de gestion. Les plans de culture étaient élaborés à Alger et aucune concertation avec les acteurs de base n'était possible.

Le seul avantage qu'ont tirés les ouvriers de ce secteur, est paradoxalement un relâchement du contrôle par rapport à la période coloniale où le colon était maître absolu des lieux et gérait son exploitation avec une rigueur imposée aux ouvriers indigènes qui ne pouvaient se permettre de discuter un ordre ou une quelconque directive.

Les dirigeants du secteur autogéré ont manifestement manipulé les chiffres et on a assisté à des distributions de bénéfices de campagne à des exploitations déficitaires, et ce uniquement pour plaire aux dirigeants politiques et conserver les privilèges acquis.

(1) Omar Bessaoud, «L'agriculture algérienne: des révolutions agraires aux réformes libérales (1963-2002)», in Du Maghreb au Proche-Orient, les défis de l'agriculture, L'Harmattan, Paris, p. 36. 
Mais, la forfaiture ne pouvait résister à la réalité de la sphère économique. Du fait de plans de campagnes inadaptés, l'offre des produits agricoles était totalement décalée par rapport aux besoins de la population. Pour les céréales, on a assisté bon an mal an à un effondrement des rendements déjà aléatoires et pour les autres productions, l'offre était plus que fantaisiste. On pouvait trouver sur les marchés, que des tomates, ou que des courgettes, ou que des pommes de terre !...

\subsection{La révolution agraire:}

A la lumière de ce constat affligeant, le président Houari Boumediene a tenté de remédier à la gabegie qui s'est installée. Dans un esprit de justice sociale, il n'a pas trouvé mieux que de proclamer une déclaration qui a fait focus par la suite. Il a pensé à juste titre que «la terre appartient à celui qui la travaille». C'est dans cette optique qu'il y a eu mise en place d'un système socialisant. L'Ordonnance 71-73 du 8 novembre 1971, a institué la révolution agraire dont le principe fut justement «La terre à celui qui la travaille». Ce qui a permis de récupérer 1,2 million ha qui étaient auparavant la propriété de grands pachas.

La loi du 8 novembre 1971 portant «Révolution agraire»a donc décidé l'extension des nationalisations au profit d'un «Fonds national de la révolution agraire» (FNRA) pour deux ensembles fonciers:

- les biens à caractères agricoles des collectivités publiques: communes, wilaya, domaine privé de l'Etat, terres de statut collectif ( $\operatorname{arch}$ ) et bien des fondations religieuses (habous);

- les biens des propriétaires agricoles qui n'exploitent pas directement et personnellement leurs terres et ceux dont les superficies excèdent un plafond déterminé.

La pratique et la réglementation ont conduit à préciser et à distinguer deux notions employées indifféremment par la loi. Celle de propriétaire non exploitant et de propriétaire absentéiste.

Lors des réformes de la décennie 80 , nombre d'anciens propriétaires qui avaient fait don de leurs terres au profit de la révolution agraire n'ont pas manqué l'occasion pour fustiger le défunt président en lui portant l'opprobre nationale. Il est utile de rappeler deux de ses déclarations qui étaient prémonitoires de ce qui est advenu par la suite à l'agriculture algérienne ${ }^{(1)}$.

(1) Omar Bessaoud, «L'agriculture algérienne: des révolutions agraires aux réformes libérales (1963-2002)», in Du Maghreb au Proche-Orient, les défis de l'agriculture, L'Harmattan, Paris, Page 40. 


\subsection{Les réformes de la décennie 80:}

Les réformes de la décennie 1980 ont voulu casser le tabou qui pesait jusque-là sur la propriété privée. Le statut des terres publiques (1987) institue un droit individuel d'exploitation et la loi d'orientation foncière qui intéresse les terres privées annule la loi de réforme agraire de 1971 et restitue des terres expropriées à leurs anciens propriétaires. C'est la réhabilitation de la propriété privée. C'est ainsi que plus de 2 millions d'hectares de bonne terre ont été cédées à des entités individuelles ou collectives avec des modes de concession qui ont évolué durant la décennie 80. Une batterie de Lois a vu le jour mais les décrets d'application tardent à être publiés et plongent ainsi ce secteur dans une opacité qui paralyse l'agriculture algérienne. L'UNPA a dénoncé ce problème en citant l'exemple d'un décret d'application de la loi $87 / 19$ qui n'a été publié que 18 ans après $! . .$.

Le résultat de ce morcellement a eu une incidence inefficace sur les plans, social et économique, dans le monde rural. Les 170277 exploitants en EAC ont de suite voulu imiter les 17632 qui ont eu la chance d'avoir bénéficié d'une concession individuelle. D'une part, des mésententes dans les EAC ont déstabilisé la production et d'autre part les bénéficiaires des EAI ont joué le jeu les premières années de la réforme et par la suite se sont mués en rentiers en louant leurs terres à des ouvriers agricoles ou à des investisseurs sans aucun rapport avec l'agriculture. Depuis 2010, une autre loi leur permet même de vendre leurs concessions. Les bénéficiaires d'EAC et EAI peuvent désormais céder des terres légalement et surtout dans un cadre qui relève de l'imposture, à la faveur de la loi 10-03 de 2010 sur les terres agricoles du domaine privé de l'Etat. Il n'y a qu'en Algérie que l'on trouve une telle turpitude.

Tableau $\mathbf{n}^{\circ} \mathbf{0 1}$. Bilan de la réorganisation des structures foncières du domaine de l'Etat (1992).

\begin{tabular}{|l|c|c|c|}
\hline Types d'exploitations & EAC & EAI & Total \\
\hline Nombre d'exploitations & 28707 & 17632 & 46339 \\
\hline Superficies (ha) & 1910109 & 222246 & 2132355 \\
\hline Nombre d'exploitants & 170277 & 17632 & 187909 \\
\hline
\end{tabular}

Source: Ministère de l'agriculture. 
L'Etat a conservé une superficie de 166234 hectares où elle a érigé 176 fermes pilotes pour 8144 salariés $^{(1)}$. Elles ont été chargées d'exécuter des programmes d'expérimentation dans le domaine des techniques de culture et d'élevage et de production de semences.

Très vite, cette réforme a montré ses limites car la notion de propriété n'a pas été perçue de la même manière par tous les intervenants du secteur agricole. Les bénéficiaires des E.A.C se sont embourbés dans des problèmes de division du travail et la production s'en est très vite ressentie.

\section{Des situations différenciées selon les zones rurales:}

L'évolution de la ruralité n'est pas homogène sur l'ensemble du territoire rural. Actuellement $42 \%$ de la population rurale vit dans des habitations éparses (5.419.525 habitants) et à plus de $58 \%$ en habitat aggloméré (6.714.401 habitants) dans près de 3500 agglomérations rurales ${ }^{(2)}$ et/ou semi rurales, cette Distribution varie selon les 48 wilayas.

Des différentiations sont à retenir dans le processus à l'intérieur des régions mais aussi entre le monde rural et le monde urbain. La localisation géographique des communes rurales (979 sur un total de 1541 soit 64\%) par grandes régions naturelles montre que le quart des communes rurales se situe dans les Hauts Plateaux, près des deux tiers (64\%) dans le Nord et enfin, $11 \%$ des communes rurales sont localisées au Sud du pays ${ }^{(3)}$. (Voir les tableaux au-dessous).

Tableau $\mathbf{n}^{\circ} \mathbf{0 2}$. Evolutions de populations (en million).

\begin{tabular}{|c|c|c|c|}
\hline Année & Rurale & Urbaine & National \\
\hline 1998 & 12.1 & 17.0 & 29.1 \\
\hline 2012 & 13.4 & 20.5 & 33.9 \\
\hline
\end{tabular}

Source: Ministère de l'agriculture.

Tableau n ${ }^{\circ}$ 03. Répartition des communes 2012.

\begin{tabular}{|l|c|c|c|c|}
\hline Communes & National & Nord & Haut plateaux & Sud \\
\hline rurale & 979 & 562 & 287 & 130 \\
\hline urbaines & 562 & 384 & 120 & 58 \\
\hline totales & 1541 & 946 & 407 & 188 \\
\hline
\end{tabular}

Source: Ministère de l'agriculture.

(1) Ministère de l'agriculture rapport annuel 2012.

(2) Office nationale des statistiques: le recensement RGPH 1998.

(3) Omar BESSAOUD LA page 80. 


\section{Le poids de l'activité agricole:}

Au regard des activités en milieu rural, la population rurale occupe à part égale dans les secteurs primaire et tertiaire, avec des taux respectivement et 39,5 et $39 \%$ des occupés et près de $25 \%$ des actifs ruraux occupe le secteur secondaire La population rurale occupée dans l'industrie représente encore $8,8 \%$ et le BTP $12,6 \%{ }^{(1)}$.

La population agricole tout en restant encore majoritaire au sein des zones rurales, évolue ainsi vers d'autres activités de services ou de transformation. La tendance à la pluriactivité des ménages ruraux accentue.

Le potentiel de main d'œuvre susceptible de s'orienter vers des activités multiples est encore important.

Plus du quart des membres des ménages sont sans emploi ou demandeurs d'emplois et la faible croissance de l'emploi rural oblige de maintenir une population sur de petites exploitations. Déjà marquée par son caractère pluvial rendant aléatoires ses performances, l'agriculture algérienne est caractérisée en effet par l'exiguïté des exploitations agricoles puisque quelque $70 \%$ de celles-ci ont une superficie inférieure à 10 hectares.

De plus, cette contrainte «structurelle» n'est compensée, ni par une politique d'extension massive des surfaces irriguées, ni par la politique de soutien à l'utilisation de facteurs d'intensification (engrais, semences de qualité...), engagée dans le cadre du programme national de développement agricole.

L'agriculture subit d'autres contraintes qui grèvent son potentiel de croissance et pèsent sur les équilibres écologiques des différentes régions naturelles. On peut citer entre autres, la surexploitation des ressources hydriques souterraines, l'érosion des sols et leur salinisation ainsi que la désertification qui menace les 32 millions hectares de terres de parcours et le couvert forestier de l'Algérie du nord. ${ }^{(2)}$

\section{Les politiques de développement agricole et rural durable:}

La stratégie du secteur a évolué d'un Plan National de Développement Agricole (PNDA), de 2000 à 2002, à un agrandissement des missions de l'agriculture au Développement rural sous la forme d'un Plan national de développement agricole et rural (PNDAR), de 2002 à 2003. Sur cette base,

(1) Office nationale des statistique. Le recensement RGPH de 1998.

(2) Omar BESSAOUD - op. cit., page 81. 
le gouvernement a préparé e2004 une Stratégie de développement rural (2004-2013) qui crée le lien entre la revitalisation des espaces ruraux et la politique de l'aménagement du territoire, par le biais d'une valorisation des activités économiques et des patrimoines naturel et humain, avec pour objectif un développement équilibré et harmonieux de tous les territoires sans exclusion ni marginalisation.

En 2006, le Conseil du Gouvernement a affirmé que la Stratégie Nationale de Développement Rural Durable et les PPDRI sont aujourd'hui une réalité et a défini les contours du concept de développement rural.

La stratégie s'articule autour de quatre axes stratégiques:

- l'établissement d'un partenariat local et d'une intégration multisectorielle au sein des territoires,

- $\quad$ un appui à la mise en œuvre d'activités économiques innovantes.

- la valorisation équilibrée et gestion durable des ressources naturelles.

- La promotion d'une synergie économique et sociale ainsi que d'une coordination efficace des actions.

- Les principaux outils pour mettre en œuvre cette stratégie sont:

- les projets de proximité de développement rural,

- les mécanismes de concertation et décision en place,

- les systèmes de programmation et d'aménagement des territoires ruraux.

- les modalités de financement des activités en milieu rural.

Ces outils concilient l'action de proximité, la synergie entre le capital naturel, Physique, financier, humain et social, et la promotion d'un partenariat associant L'ensemble des acteurs concernés à la mise en œuvre des programmes et actions Retenus. ${ }^{(1)}$

\section{- $\quad$ Les trois piliers de la politique de Renouveau Agricole et Rural:}

La politique de Renouveau Agricole et Rural, instrument pour la concrétisation de la souveraineté alimentaire, se décline en trois volets complémentaires:

- Le Renouveau Agricole.

- $\quad$ Le Renouveau Rural.

- Le Renforcement des Capacités Humaines et de l'Appui Technique aux Producteurs. ${ }^{(2)}$

\section{9- Réalisations et projets:}

Les réalisations au plan agricole induites par les investissements engagés, il a été enregistré l'identification de plus de:

(1) CNERU- SDAAM op. cit., page 493-494.

(2) Ministère de l'agriculture et de développement rural - la politique de renouveau agricole et rural en Algérie- rapport annuel 2010 page 1-2. 
- 2000 projets de mise en valeur de proximité au niveau des 48 wilayas du pays, la validation de 480 projets par les comités techniques de wilaya dont 80 projets approuvés par les walis parmi lesquels 35 projets ont bénéficié de décisions de financement partiel sur le fonds de développement rural et de mise en valeur des terres par la concession (FDRMVCT). En termes d'impact sur les populations, les projets identifiés fin 2002 concernent plus de 130.000 ménages soit quelque 700.000 personnes, réparties sur l'ensemble du territoire national.

- L'accroissement de la production nationale en produits de large consommation (blé dur, lait) assurant un taux de couverture moyen minimal de $75 \%$ des besoins;

- La modernisation et la diffusion des progrès technologiques dans les exploitations agricoles (irrigation adaptée, fertilisation, mécanisation, utilisation des semences et géniteurs améliorés...).

- La modernisation et l'organisation des réseaux de collecte et de commercialisation de la production nationale et d'approvisionnement en intrants et services à l'agriculture

- La mise en place des systèmes de régulation interprofessionnels, fédérant les différents maillons des filières de large consommation (SYRPALAC) tels que les céréales, lait, pomme de terre, oléiculture, viandes ...etc créant les conditions déstabilisation des marchés.

- La généralisation et l'extension des systèmes d'irrigation agricole en visant 1,6millions d'hectares, à l'horizon 2014, contre près de 900.000 hectares actuellement.

- Le développement des capacités nationales pour atteindre l'autosuffisance dans la couverture des besoins en matière de semences, plants et générateur d'un développement des espaces ruraux équilibrés, harmonieux et durables.

\section{0- Les objectifs et les perspectives:}

Il a été attendu de ce programme en l'an 2014, l'amélioration de la sécurité Alimentaire de l'Algérie induite par les effets conjugués:

- D'une amélioration du taux de croissance de la production agricole moyenne passant d'un niveau moyen de 6\% par an (période 20002008) à 8,33\% sur la période 2010-2014.

- De l'accroissement de la production nationale et l'amélioration de son intégration et de sa collecte: La stabilisation de la production céréalière à 54 millions de quintaux (réduction des importations d'orge et de blé dur) et celle du lait à plus de 3 milliards de litres avec un milliard de litres collecté (à intégrer pour répondre aux besoins en laits pasteurisés) devrait influer aussi de manière effective sur le niveau des importations de la poudre de lait. 
- Du renforcement d'un développement durable et équilibré des territoires et amélioration des conditions de vie des populations rurales: à travers 10.200 projets de proximité de développement rural intégré (PPDRI) dans 2.174 localités rurales.

- Ils permettront d'améliorer les conditions de vie de 727000 ménages ruraux soit près de 4471000 habitants et d'avoir un impact sur la préservation et la valorisation de 8,2 millions d'hectares situés dans les zones de montagnes, les espaces steppiques et les zones sahariennes.

- De la relance durable de l'appareil industriel national et l'amélioration de l'intégration agro-industrielle: Les mesures mises en place dans le cadre du renouveau agricole en matière de soutien à l'intensification des filières auront des effets importants sur le développement d'une industrie agricole et d'un réseau de petites et moyennes entreprises de production d'intrants et de services à l'agriculture notamment pour répondre aux besoins importants générés par le programme La politique de renouveau agricole et rural en Algérie ${ }^{(1)}$.

- De la création d'emploi: La création de près de 1200000 équivalents emplois permanents, notamment dans des zones pauvres en opportunités de création d'emplois et de création de revenus hors agriculture.

\section{1- Étude impacte du Renouveau Agricole et Rurale:}

\section{1-1- Le Renouveau Agricole:}

- La modernisation et à l'intensification des filières de production de large consommation à travers les soutiens à la mécanisation, à la fertilisation aux systèmes d'irrigation, semences, plants et géniteurs .... Pour les filières Céréales, lait, pomme de terre, viandes.... :

- L'incitation directe à la production pour les céréales, le lait, les semences...

- Le développement des systèmes de régulation et la protection des revenus des Agriculteurs notamment pour la filière pomme de terre et son extension progressives aux autres produits agricoles.

- La réalisation et la valorisation des infrastructures de stockage et de logistique sous froid (08 millions de mètres cubes).

- L'appui à la réalisation de 39 silos de stockage. ${ }^{(2)}$

\section{1-2- Le renouveau rural:}

Les 10200 projets prévus au titre des contrats de performance de renouveau rural pour la période 2010-2014 se caractérisent par les indicateurs d'impacts suivants:

(1) Ministère de l'agriculture et de développement agricole - op. cit., page 5-6.

(2) Op. cit., page 6-7. 
- La protection des bassins -versants de barrages:

Il porte sur l'application des études réceptionnées définitivement pour 34 bassins- versants sur une superficie de3,5 millions d'ha localisés au niveau de 25 wilayas et concernent plus de 350communes dont la population recensée est de 07 millions d'habitants.

- Le programme de lutte contre la désertification:

Il est prévu sur 30 wilayas et 338 communes. Il vise un espace de 2,5 millions d'ha dont 137000 ha seront traités.

- La réhabilitation et l'extension du patrimoine forestier: Il a pour objectif Notamment l'amélioration de l'état et de la productivité des peuplements forestiers (la réalisation des travaux sylvicoles sur $118500 \mathrm{Ha}$ et la plantation sur 36000 ha.

- Le programme de Conservation des Ecosystèmes Naturels: Il est attendu que ce programme touche 52 communes et 35000 ménages ruraux, le traitement d'un espace sur $188000 \mathrm{Ha}$ et la création de 17 500 équivalent emplois permanents.

- L'extension de la SAU pour la mise en valeur des terres agricoles: La Programmation des projets prévus concernant la mise en valeur par la concession de près de 230.000 ha.

\section{1-3- Le Renforcement des Capacités Humaines et de l'Appui Technique aux producteurs:}

Pour cela, le programme prévoit la mobilisation d'une enveloppe annuelle de 24 milliards de dinars par an consacrée aux principales actions de renforcement suivantes:

- $\quad$ La mise en œuvre de programme d'envergure de perfectionnement, de formation et de démonstration des technologies agricoles au bénéfice des Agriculteurs et des opérateurs.

- La réhabilitation et la construction de nouvelles stations expérimentales et de Laboratoires des instituts techniques de développement par filière du secteur.

- La réhabilitation des centres de formation et d'apprentissage du secteur.

- Le renforcement de l'encadrement technique et des services spécialisés et de Leur présence territoriale (service d'appui technique, services phytosanitaires Vétérinaires, forestiers ...).

- Le renforcement de l'administration locale et des moyens de suivi et de contrôle des programmes, y compris les systèmes d'information et de communication.

- Le développement d'études et la mobilisation des compétences nationales et Universitaires autour des problématiques du développement agricole et rural Et de l'appui en matière d'expertise pour la mise en ouvre des projets. 
En effet, et dans le contexte du développement agricole, l'état Algérien a met en œuvre à travers le Schéma National de l'Aménagement du Territoire (SNAT) horizons 2025, un programme d'actions territoriales afin d'assurer l'équité territoriale en milieu rural à travers l'égalité des chances d'accès pour l'ensemble des citoyens du territoire national à un logement répondant aux moyens d'hygiène, eau, assainissement, énergie, sécurité, à l'emploi, aux services publics notamment les transports, à la santé, à l'éducation et la formation. rural:

Ce programme a met quatre conditions essentielles au développement

\section{- Définir la politique agricole}

Clarifier une confusion: le développement rural passe par l'agriculture, mais également par d'autres activités: d'où la tendance à rechercher le salut dans et hors agriculture. Les activités peuvent se développer en s'appuyant en partie sur le pilier agricole et sur l'ouverture à d'autres secteurs.

- Définir le contenu de la multi activités: en prolongement naturel du développement agricole.

- Les accompagner par une politique d'équipements et de services dynamiques:

- eau, santé, éducation, énergie.

- transport, télécommunication, communication, services, logements. Cette politique devra impliquer des acteurs organisateurs. Il faudra dégager une vision agricole qui intègre les petites villes avec leur environnement et en tant que centre d'animation et d'impulsion et d'équipement pour le rural, les espaces naturels, les massifs et la steppe.

- Inscrire le monde rural dans des relations urbaines-rurales denses et complémentaires:

La problématique du développement rural inclut ici la liaison entre le monde rural et les petites agglomérations. Il s'agit de faire en sorte que ces agglomérations soient des centres d'animation et de rayonnement économique

- $\quad$ Renforcement des bases productives et diversification rurale:

- Mise à niveau des infrastructures et des équipements de base amélioration de la productivité des systèmes de culture et d'élevage.

- Développement d'une industrie agro-alimentaire valorisant les productions locales de l'agriculture et de l'élevage. 
- Développement de la commercialisation des produits.

- Diversification de l'économie rurale par le développement d'activités valorisant des ressources naturelles, patrimoniales et humaines.

- Renforcement des complémentarités et des relations urbain - rural: Les solidarités entre monde urbain et monde rural sont valorisées.

- $\quad$ Les Projets de Proximité du Développement Rural Intégrés "PPDRI":

Les PPDRI sont des projets intégrés fédérateurs construits de bas en haut dans la responsabilité partagée entre les services de l'administration, les élus locaux les citoyens et les organisations rurales Ils mutualisent autour de thèmes fédérateurs les efforts des acteurs publics et privés pour la réalisation d'investissements à usage collectif financés sur les budgets des secteurs, des wilayas et des communes, des PCD et des investissements à usage individuel portés par des personnes physiques, soit autofinancés, soit faisant appel aux différents dispositifs de soutien de l'investissement.

Thèmes fédérateurs autour desquels seront construits les PPDRI:

* Modernisation et/ou réhabilitation d'un village et de petites agglomérations amélioration de la qualité et des conditions de vie en milieu rural.

* Diversification des activités économiques en milieu rural (tourisme rural artisanat, produits locaux, valorisation des sites culturels, PME/PMI, énergies renouvelables, Technologies de l'Information et de la Communication ...) et amélioration de l'attractivité du territoire rural (jeunes, retour des populations Nouvelles installations....).

* Protection et valorisation des ressources naturelles (forêt, steppe, oasis montagne, littoral).

* Protection et valorisation du patrimoine rural matériel ou immatériel (produits du terroir, bâtis, préservation des sites et des produits historiques et culturels valorisation des manifestations traditionnelles).

Dans une wilaya, plusieurs PPDRI regroupés par thème fédérateur constituent alors l'un des quatre programmes par objectifs de développement rural intégré de la wilaya. L'agrégation des quatre Programmes par objectifs constitue le Programme de Développement Rural Intégré de la Wilaya «PDRIW». Les PDRIW constituent le Plan de Soutien au Renouveau Rural PSRR. 


\section{- Les instruments de financement:}

La diversité des porteurs de projet ruraux (taille, revenus, capacités d'autofinancement etc.) met en évidence la nécessité d'adapter la politique de soutien de l'Etat et de promouvoir des systèmes de financement adaptés. De nouveaux systèmes de financement sont proposés en plus des offres du système bancaire classique, notamment en matière de micro finance. Ces systèmes seront fondés sur la promotion:

* Du micro-crédit.

* De systèmes d'assurances économiques et sociales.

* D’organismes de garantie des prêts.

* De banques communautaires.

* De mutuelles ou de coopératives d'épargne ou de crédit.

* De formes de cautions mutuelles et solidaires.

- L'adaptation de la politique de financement en milieu rural: Le soutien à l'investissement en milieu rural place le crédit comme élément central du processus de mise à niveau de l'agriculture et d'amélioration de ses performances et la diversification économique dans les espaces ruraux.

\section{Conclusion}

Tous les programmes et plans de développement agricole sont confrontés à des défis majeurs en matière de sécurité alimentaire visant à améliorer le niveau de vie de la population rurale et à réduire la migration des campagnes vers la ville. Les résultats restent l'otage de ceux qui les mettent en œuvre et les reflètent sur le terrain., Et il peut être dit que la stratégie nationale pour le développement agricole durable, même si elle n'atteint pas les objectifs, mais il a obtenu des résultats positifs dans une large mesure.

\section{Bibliographie}

1. AZZI Malika - Evaluation multicritères de la durabilité agro écologique et socio territoriale des exploitations agricole dans la Mitidja - mémoire d'ingénieur d'état en agronomie -école nationale supérieure agronomique el Harrach- Alger

2. BIEWINGA, E.E., VAN DER BIJL, G. (1996). Sustainability of energy crops. A methodology developed and applied, Report no. 234. Centre for Agriculture and Environment (CLM), Utrecht, The Netherlands. (inSrour. 2006).

3- MYRDAL, «Planifier pour développer. De l'Etat providence au monde providence», 1960, cité par Y. HAYAMI et V.W. RUTTAN, «Agriculture et développement, approche internationale», Edition : INRA, Paris, 1998. 
4. M. BIALES, R, Leurion, J-L RIVAUD, «Notions fondamentale d'économie», 3ème Edition : Foucher, Paris, 2002.

5- Omar Bessaoud IAMM « Les changements climatiques et les risques sur l'agriculture ». El watan Economie. Alger. Juillet 2008.

6- Omar Bessaoud, «L'agriculture algérienne: des révolutions agraires aux réformes libérales (1963-2002)», in Du Maghreb au Proche-Orient, les défis de l'agriculture, Le Harmattan, Paris.

7- Ministère de l'agriculture et de développement rural: le stratège de développement rural durable rapport 2012.

8- Schéma National de l'Aménagement du Territoire. Horizons 2025. 